\title{
Peristalsis by pulses of activity
}

\author{
N. Gorbushin and L. Truskinovsky \\ PMMH, CNRS-UMR 7636, CNRS, ESPCI Paris, PSL Research University, 10 Rue Vauquelin, 75005 Paris, France
}

(Received 5 December 2020; accepted 23 March 2021; published 15 April 2021)

\begin{abstract}
Peristalsis by actively generated waves of muscle contraction is one of the most fundamental ways of producing motion in living systems. We show that peristalsis can be modeled by a train of rectangular-shaped solitary waves of localized activity propagating through otherwise passive matter. Our analysis is based on the Fermi-Pasta-Ulam (FPU) type discrete model accounting for active stresses and we reveal the existence in this problem of a critical regime which we argue to be physiologically advantageous.
\end{abstract}

DOI: 10.1103/PhysRevE.103.042411

\section{INTRODUCTION}

Peristalsis is a series of actively generated wavelike muscle contractions and relaxations which propagate along the body of an organism. Smooth muscle tissues develop such contractions to produce a peristaltic wave in the digestive tract [1,2]. Crawling by peristalsis enables organisms like snails, earthworms, slugs, and terrestrial planarians to advance in narrow spaces [3-8]; based on geometrical symmetries only, peristaltic waves were shown to be an optimal motility strategy in such systems [9].

In this paper we develop a prototypical model of a peristalsis in a segmented limbless organism. We assume that it crawls along a flat surface by extending its forward end and then bringing up its rear end. To achieve this goal the organism generates a solitary wave which travels from the front to the rear. The space-time distribution of activity during peristalsis is known to be highly adaptive [10] and the mechanism of this adaptability is a subject of great interest in robotics $[11,12]$.

Peristaltic waves are also of general interest as elementary nonlinear excitations of active matter. Propagating active pulses reminiscent of peristaltic waves are ubiquitous in nature, from shimmering in honeybees [13] to spectator waves in stadiums [14]. Comparable phenomena in the form of propagating activity bands are also observed in flocking colonies of swarming robots and other similar systems [15,16].

Some of these behaviors can be quantified using models of excitable media [17] or models involving globally synchronized actuation [18]. However, such models have been questioned in cases clearly dominated by mechanical sensory feedback and neuromechanical proprioception [19]. In view of distinctly mechanical nature of peristalsis, we forgo the reaction-diffusion framework [20] and disregard the possibility of centralized control [21]. Instead, we assume that physical forces not only drive the associated localized waves of activity but also secure the signaling pathways regulating the crucial internal delays.

As a minimal model, capturing only the main effect, we consider a mass-spring chain capable of generating active stresses. Behind such activity is an intricate endogenous $m a$ chinery of the type involved in muscle tetanization. However, following the approach of active gel theory [22], we leave it outside our model and assume that the associated energy flow through the system can be represented by a nonconstitutive component of stress. We show that the ensuing, apparently purely mechanical, model can generate directional peristaltic locomotion without relying on externally coordinated actuators or digital controllers. Our intentionally minimalistic approach emulates (and can be extended towards) more comprehensive continuum theories of activity in both fluids [22,23] and solids [24-27]. While more comprehensive models of active media directly account for energy inflow and the compensating dissipation, in our simplified model we assume that both flows match and therefore can be neglected.

Passive solitary waves have been long employed in actuator-driven soft robotics imitating peristalsis [28-30]. Instead, here we rely on self-driven active solitary waves and show that peristalsis can be modeled by a train of such waves propagating through otherwise passive matter. Rather remarkably, our analysis reveals the existence of a critical motility regime around which the active pulses assume realistic rectangular shape whose width can vary broadly. This ensures a wide-ranging repertoire of macroscopic responses and we argue that the so-interpreted criticality may be a characteristic feature of the physiological peristalsis.

The paper is organized as follows. In Sec. II we introduce our main modeling assumptions. A quasicontinuum approximation of the original discrete model is studied in Sec. III. The obtained results are used in Sec. IV to construct a model of peristaltic motion. We return to the discrete model in Sec. V and show that in this case the problem can be also solved explicitly. Our conclusions are summarized in Sec. VI.

\section{THE MODEL}

Bodies of annelid animals are usually divided into a series of metameres, the segments that are fundamentally similar in muscular structure and functionality [7,31]. To model such organisms we first neglect friction [32-34], and represent them schematically as a chain of springs connected in series. 
(a)

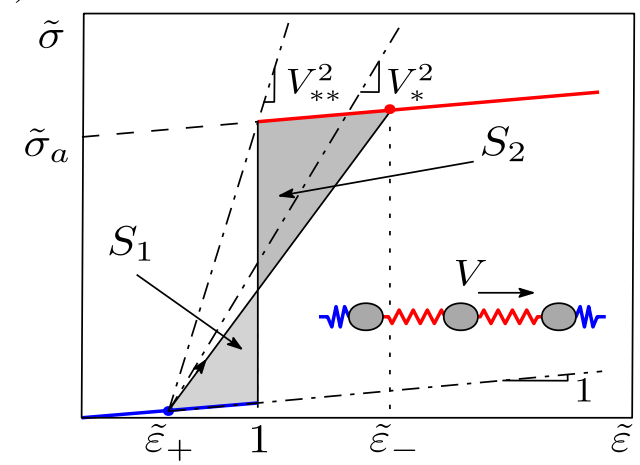

(b)

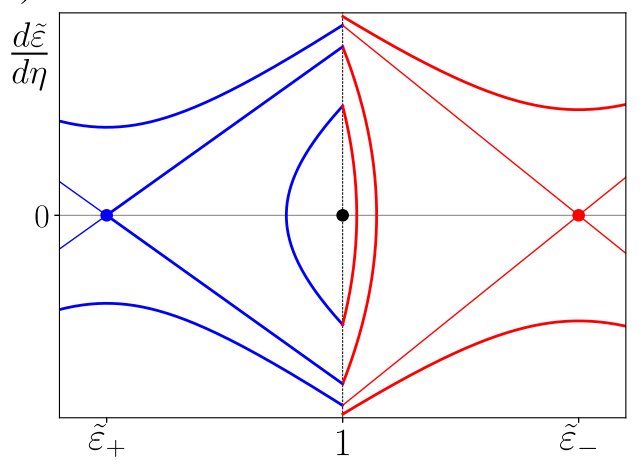

FIG. 1. (a) Stress-strain relation with passive (blue) and active (red) branches. The kink, connecting $\tilde{\varepsilon}_{ \pm}$at the critical value of velocity $V=V_{*}$ when $S_{2}=S_{1}$, is dissipation free. (b) Phase portrait of the continuum system (3) at $1<V<V_{*}$. The extension pulse corresponds to the homoclinic trajectory starting and ending at $\tilde{\varepsilon}_{+}$; contraction pulse, which exists at $V_{*}<V<V_{* *}$, is a homoclinic trajectory starting and ending at $\tilde{\varepsilon}_{-}$(not shown).

The dynamics of such system is described by the Fermi-PastaUlam (FPU) equations [33]

$$
\rho a^{2} \frac{\partial^{2} \varepsilon_{n}}{\partial t^{2}}=\sigma\left(\varepsilon_{n+1}\right)+\sigma\left(\varepsilon_{n-1}\right)-2 \sigma\left(\varepsilon_{n}\right)
$$

where $\varepsilon_{n}(t)=\left(u_{n+1}(t)-u_{n}(t)\right) / a$ are the strains in the springs, $u_{n}(t)$ are the displacements of the nodes, and $a$ is the equilibrium length. The inertial term, allowing the system to overcome the discreteness-induced trapping, proved to be important in ultrasoft robotics $[30,31,35,36]$. In physiological setting the apparent mass density $\rho$ can be viewed as a parameter introducing the activity-related time delay in the response of stretch receptors [37,38].

We assume that the constitutive relation for the stress $\sigma$ has two branches: passive and active; see Fig. 1(a). For simplicity, the soft elastic response along the passive branch is considered linear $\sigma=E \varepsilon$, with elastic modulus $E$. To describe the active branch (analog of muscle tetanus), we write $\sigma=\sigma_{a}+E \varepsilon$, where $\sigma_{a}>0$ is a constant active stress; in more detailed models it can be a variable with sigmoidal response taking a value zero in the passive phase [19]. We further assume that switching from passive to active response takes place when the "unjamming" threshold strain $\varepsilon_{c}$ is reached [38]. We neglect the possibility of hysteresis and assume that unloading below the threshold $\varepsilon_{c}$ brings the system back into the passive state; see Fig. 1(a). To nondimensionalize the system (1) we normalize length by the system size $L$, time by $L / c$, where $c=\sqrt{E / \rho}$, and stress by $E$.

\section{QUASICONTINUUM APPROXIMATION}

It will be convenient to first deal with a quasicontinuum approximation of the discrete problem. To this end we introduce the continuous strain field $\varepsilon(x, t)$, where $\varepsilon(n h, t)=\varepsilon_{n}(t)$, and assume that $h=a / L \ll 1$; we will also use the convenient rescaling $\tilde{u}=u / \varepsilon_{c}, \tilde{\varepsilon}=\varepsilon / \varepsilon_{c}$, and $\tilde{\sigma}=\sigma / \varepsilon_{c}$. If we now Padé approximate the nonlocal operator in the right-hand side of (1) and leave only the lowest order terms, we obtain [39-41]

$$
\left(1-\frac{h^{2}}{12} \frac{\partial^{2}}{\partial x^{2}}\right) \frac{\partial^{2} \tilde{\varepsilon}}{\partial t^{2}}=\frac{\partial^{2} \tilde{\sigma}}{\partial x^{2}} .
$$

To generate a solitary wave solution of (2) we impose a traveling wave ansatz $\varepsilon(x, t)=\varepsilon(\eta)$, where $\eta=(x-V t) / h$ and $V$ is the dimensionless velocity of the pulse.

If we center the active pulse performing local extension at $\eta=0$ and denote its width by $2 d$ we can write the associated stress distribution in the form $\tilde{\sigma}(\eta)=\tilde{\varepsilon}(\eta)+$ $\tilde{\sigma}_{a} \operatorname{rect}(\eta /(2 d))$, where $\operatorname{rect}(x)=H(x+1 / 2)-H(x-1 / 2)$ and $H(x)$ is the Heaviside function. We require that $\tilde{\varepsilon}(\eta) \rightarrow 0$ as $\eta \rightarrow \pm \infty$ and impose at $\eta= \pm d$ the matching conditions $\llbracket \tilde{\varepsilon} \rrbracket=\llbracket d \tilde{\varepsilon} / d \eta \rrbracket=0$ and set $\tilde{\varepsilon}( \pm d)=1$. Then, integrating (2) twice and applying the boundary and matching conditions, we obtain the equation

$$
\left(V^{2}-1-\frac{V^{2}}{12} \frac{d^{2}}{d \eta^{2}}\right) \tilde{\varepsilon}=\tilde{\sigma}_{a} \operatorname{rect}\left(\frac{\eta}{2 d}\right),
$$

where the right-hand side implicitly depends on $\tilde{\varepsilon}$. A similar solitary wave solution, describing local contraction, can be obtained if we set $\tilde{\sigma}(\eta)=\tilde{\varepsilon}(\eta)+\tilde{\sigma}_{a}[1-\operatorname{rect}(\eta /(2 d))]$ and require that $\tilde{\varepsilon} \rightarrow \lambda$ when $\eta \rightarrow \pm \infty$, where

$$
\lambda=\tilde{\sigma}_{a} /\left(V^{2}-1\right) .
$$

The extension pulses exist in the range $1<V<V_{*}$ where $V_{*} \equiv \sqrt{\tilde{\sigma}_{a} / 2+1}$, so they are supersonic, which does not mean that they are fast given that the underlying elastic medium is almost an acoustic vacuum [42]. The phase portrait of the system (3) at $1<V<V_{*}$ is shown in Fig. 1(b). Two nondegenerate saddle points at $\tilde{\varepsilon}_{ \pm}$lie on the same Rayleigh line $V^{2}=\left[\tilde{\sigma}(\tilde{\varepsilon})-\tilde{\sigma}\left(\tilde{\varepsilon}_{+}\right)\right] /\left(\tilde{\varepsilon}-\tilde{\varepsilon}_{+}\right)$; see Fig. 1(a). Solitary waves describing the extension pulses correspond to homoclinic trajectories starting and ending at $\tilde{\varepsilon}_{+}$. Periodic trains of such pulses correspond to closed trajectories encircling the degenerate center at $\tilde{\varepsilon}=1$. As $V \rightarrow V_{*}$ homoclinic trajectories become heteroclinic and the solitary waves turn into kinks; at $V=V_{*}$ we have $S_{1}=S_{2}$ in Fig. 1(a) and therefore the associated macroscopic discontinuity is dissipation free [43]. The structure of these solutions is similar to the one in flocking models $[15,44]$ modulo the fact that here we omit the explicit description of inflow and outflow of energy.

The contraction pulses, exist in the complimentary range of parameters $V_{*}<V<V_{* *}$, where $V_{* *} \equiv \sqrt{\tilde{\sigma}_{a}+1}>V_{*}$. They 
correspond to homoclinic trajectories starting and ending at $\tilde{\varepsilon}_{-}$.

It is natural to start the analysis with kinks that are characterized by a single transition event at $\eta=0$. The corresponding equation reads

$$
\left(V^{2}-\frac{V^{2}}{12} \frac{d^{2}}{d \eta^{2}}\right) \tilde{\varepsilon}(\eta)=\tilde{\sigma}(\eta)+\tilde{\varepsilon}_{+},
$$

where $\tilde{\sigma}(\eta)=\tilde{\varepsilon}(\eta)+\tilde{\sigma}_{a} H(-\eta)$. The integration of this equation gives

$$
\tilde{\varepsilon}_{k}(\eta)= \begin{cases}\tilde{\varepsilon}_{+}+\frac{\lambda}{2} e^{-\eta / z}, & \eta>0 \\ \tilde{\varepsilon}_{-}-\frac{\lambda}{2} e^{\eta / z}, & \eta<0\end{cases}
$$

where

$$
z=V / \sqrt{12\left(V^{2}-1\right)}
$$

and $\tilde{\varepsilon}_{-}=\tilde{\varepsilon}_{+}+\lambda$. Imposing the matching condition $\tilde{\varepsilon}(0)=1$ we obtain the closure relation $\tilde{\varepsilon}_{ \pm}=1 \mp \lambda / 2$, which is equivalent to the requirement that $V=V_{*}$ and that the two areas in Fig. 1 are equal: $S_{1}=S_{2}$. Integration of the equation $\tilde{\varepsilon}_{k}(\eta)=$ $d \tilde{u}_{k}(\eta) / d \eta$ allows one to reconstruct the displacement field

$$
\tilde{u}_{k}(\eta)= \begin{cases}\tilde{\varepsilon}_{+} \eta-\frac{\lambda z}{2} e^{-\eta / z}, & \eta>0, \\ \tilde{\varepsilon}_{-} \eta+\frac{\lambda z}{2} e^{\eta / z}, & \eta<0 .\end{cases}
$$

Extension solitary wave solutions must satisfy

$$
\left(V^{2}-\frac{V^{2}}{12} \frac{d^{2}}{d \eta^{2}}\right) \tilde{\varepsilon}(\eta)=\tilde{\sigma}(\eta)
$$

where $\tilde{\sigma}(\eta)=\tilde{\varepsilon}(\eta)+\tilde{\sigma}_{a} \operatorname{rect}\left(\frac{\eta}{2 d}\right)$. Equation (7) should be supplemented by the condition $\tilde{\varepsilon}_{ \pm}=0$. The active stress $\tilde{\sigma}_{a}$ is now applied on the finite interval $2 d$ and we should integrate the above equation under the assumption that $\tilde{\varepsilon}( \pm d)=1$. As we have already mentioned, solution of this problem exists in the interval $1<V \leqslant V_{*}$. The strain can be written explicitly:

$$
\tilde{\varepsilon}(\eta)= \begin{cases}e^{-(\eta-d) / z}, & \eta>d, \\ \lambda+(1-\lambda) \frac{\cosh (\eta / z)}{\cosh (d / z)}, & -d<\eta<d, \\ e^{(\eta+d) / z}, & \eta<-d .\end{cases}
$$

To obtain the displacement field $\tilde{u}(\eta)$ we again set the trivial integration constant to 0 by assuming that $\tilde{u}(\eta) \rightarrow 0$ when $\eta \rightarrow \infty$ and we get

$$
\tilde{u}(\eta)= \begin{cases}-z e^{-(\eta-d) / z}, & \eta>d, \\ \lambda(\eta-d)+z(1-\lambda) \frac{\sinh (\eta / z)}{\cosh (d / z)}, & -d<\eta<d, \\ -\Delta \tilde{u}+z e^{(\eta+d) / z}, & \eta<-d .\end{cases}
$$

To close the system we must impose the continuity of the derivative $d \tilde{\varepsilon} / d \eta$ at $\eta= \pm d$. We can then compute the remaining constants

$$
d=-z \tanh ^{-1}\left(\frac{1}{1-\lambda}\right)
$$

and $\Delta \tilde{u}=2 d \lambda$. Defining the amplitude of the pulse as $A=$ $\max \tilde{\varepsilon}(\eta)-\min \tilde{\varepsilon}(\eta)$, we find for extension pulses that

$$
A=\lambda+(1-\lambda) / \cosh (d / z)
$$

To obtain contraction pulses we need to solve the equation

$$
\left(V^{2}-\frac{V^{2}}{12} \frac{d^{2}}{d \eta^{2}}\right) \tilde{\varepsilon}(\eta)=\tilde{\sigma}(\eta),
$$

where $\tilde{\sigma}(\eta)=\tilde{\varepsilon}(\eta)+\tilde{\sigma}_{a}\left[1-\operatorname{rect}\left(\frac{\eta}{2 d}\right)\right]$. The boundary conditions must be now chosen in the form $\tilde{\varepsilon} \rightarrow \lambda$ when $\eta \rightarrow \pm \infty$.

As we know, solution of this problem exists in the interval $V_{*}<V \leqslant V_{* *}$. It can be again written explicitly for the strain field

$$
\tilde{\varepsilon}(\eta)= \begin{cases}\lambda+(1-\lambda) e^{-(\eta-d) / z}, & \eta>d, \\ \frac{\cosh (\eta / z)}{\cosh (d / z)}, & -d<\eta<d, \\ \lambda+(1-\lambda) e^{(\eta+d) / z}, & \eta<-d,\end{cases}
$$

and for the displacement field

$$
\tilde{u}(\eta)= \begin{cases}\lambda(\eta-d)-z(1-\lambda) e^{-(\eta-d) / z}, & \eta>d, \\ z \frac{\sinh (\eta / z)}{\cosh (d / z)}, & -d<\eta<d, \\ \Delta \tilde{u}+\lambda(\eta-d)+(1-\lambda) z e^{(\eta+d) / z}, & \eta<-d .\end{cases}
$$

The remaining parameters are

$$
d=-z \tanh ^{-1}(1-\lambda), \quad A=\lambda-\frac{1}{\cosh (d / z)},
$$

and $\Delta \tilde{u}=2 d \lambda$.

This solution degenerates in two limiting cases. First, at $V=1$ the integration of (7) gives $\tilde{\varepsilon}(\eta)=6 \tilde{\sigma}_{a}\left(d^{2}-\eta^{2}\right)+1$ when $-d<\eta<d$ and $\tilde{\varepsilon}(\eta)=1$ when $|\eta|>d$. Imposing the continuity of derivatives at $\eta= \pm d$, we obtain $d=0$ and $A=0$ and, therefore, $\tilde{\varepsilon}(\eta) \equiv 1$ and $\tilde{\sigma}(\eta) \equiv 1$. In the other singular limit $V=V_{* *}$ we have $\lambda=1$ and therefore from (13) we find that $\tilde{\varepsilon}=1$ for $|\eta|>d$. The continuity of strain derivatives at $\eta= \pm d$ again gives $d=0$ and $A=0$, hence $\tilde{\varepsilon}(\eta) \equiv 1$ but now $\tilde{\sigma}(\eta) \equiv 1+\tilde{\sigma}_{a}$.

The behavior functions $d(V)$ and $A(V)$ for both types of pulses is shown in Figs. 2(a) and 2(b). The two families are separated by the critical value of parameter $V=V_{*}$, where the solitary waves take the form of infinitely separated kinks. At this point the parameter $d$, playing the role of the correlation length, diverges even though the pulse amplitude remains finite (taking the value $A=2$ ). In the limits $V \rightarrow 1$ and $V \rightarrow V_{* *}$ we obtain sonic waves in passive and active states, respectively; note that the passive limit is singular. The typical functions $\tilde{\varepsilon}(\eta)$ for different values of $V$ are shown in the insets in Fig. 2. We emphasize that only the near-critical pulses have a physiologically realistic rectangular form.

Next, recall that, in the case of a extension pulse, the amplitude of the displacement increment, culminating the passing of a pulse, is $\Delta \tilde{u}=2 d \lambda$. As a rough description of a peristaltic wave train, represented by a succession of $N$ such pulses, we can write $\Delta \tilde{u}=2 d N \lambda$. To obtain the actual solutions describing the trains of extension pulses we need to solve the equation

$$
\left(V^{2}-\frac{V^{2}}{12} \frac{d^{2}}{d \eta^{2}}\right) \tilde{\varepsilon}(\eta)=\tilde{\sigma}(\eta)
$$

where $\tilde{\sigma}(\eta)=\tilde{\varepsilon}(\eta)+\tilde{\sigma}_{a} \sum_{j} \operatorname{rect}\left(\frac{\eta_{j}}{2 d_{p}}\right)$ and $\eta_{j}=\eta-2 j D$. Here it was assumed that each pulse has a half-width $d_{p}$ 
(a)

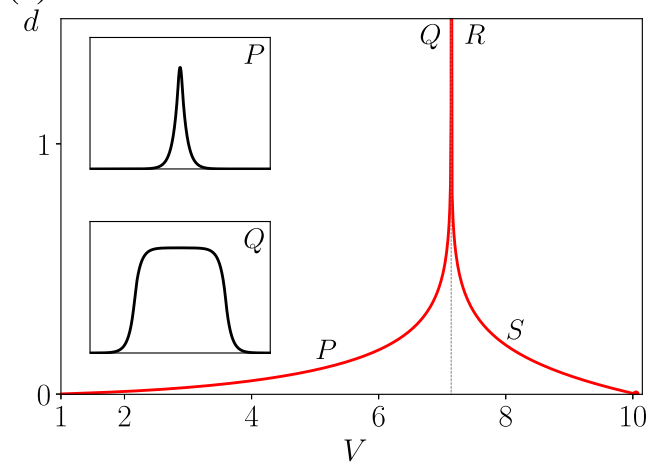

(b)

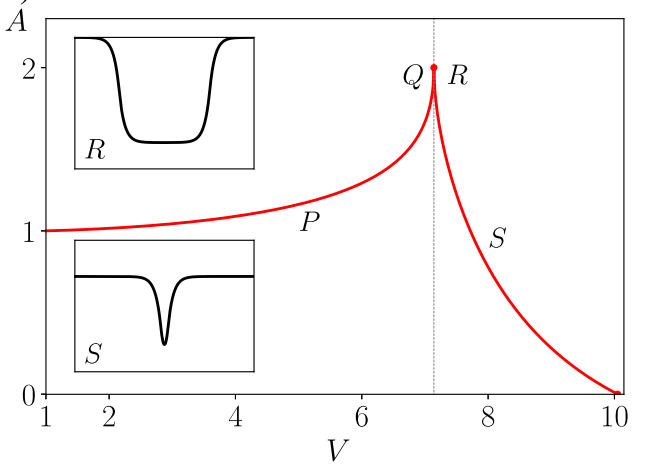

FIG. 2. Parametric dependence of the structure of active pulses at $\tilde{\sigma}_{a}=100$ : (a) the half-width $d=d(V)$; (b) the amplitude $A=A(V)$; insets show the strain profiles at $V=5$ (point $P$ ), $V=V_{*} \pm \delta$, where $\delta=10^{-7}$ (points $Q$ and $R$ ), and $V=8$ (point $S$ ). Vertical asymptotes mark the location of the critical point $V=V_{*}$.

and the whole active lattice has the period $2 D$. The matching conditions are now $\llbracket \tilde{\varepsilon} \rrbracket=\llbracket d \tilde{\varepsilon} / d \eta \rrbracket=0$ and $\tilde{\tilde{\varepsilon}}\left(j D \pm d_{p}\right)=1$.

Integration of (16) together with the matching conditions gives

$$
\tilde{\varepsilon}(\eta)= \begin{cases}\frac{e^{d_{p} / z}}{1+e^{2\left(d_{p}-D\right) / z}}\left(e^{\left(\eta_{j}-2 D\right) / z}+e^{-\eta_{j} / z}\right), & d_{p}<\eta_{j} \leqslant D, \\ \lambda+(1-\lambda) \frac{\cosh \left(\eta_{j} / z\right)}{\cosh \left(d_{p} / z\right)}, & -d_{p}<\eta_{j}<d_{p}, \\ \frac{e^{d_{p} / z}}{1+e^{2\left(d_{p}-D\right) / z}\left(e^{\eta_{j} / z}+e^{-\left(\eta_{j}+2 D\right) / z}\right),} & -D<\eta_{j}<-d_{p} .\end{cases}
$$

The value of the parameter $d_{p}$ can be found as a positive real root of the transcendental equation

$$
(1-\lambda) \tanh \left(d_{p} / z\right)=\tanh \left[\left(d_{p}-D\right) / z\right]
$$

which ensures the continuity of the first derivative of strain at $\eta_{j}= \pm d_{p}$. By integrating (17), while respecting the continuity of displacements, we obtain

$$
\tilde{u}(\eta)= \begin{cases}j \Delta \tilde{u}+\frac{z e^{d_{p} / z}}{1+e^{2\left(d_{p}-D\right) / z}}\left(e^{\left(\eta_{j}-2 D\right) / z}-e^{-\eta_{j} / z}\right), & d_{p}<\eta \leqslant D, \\ j \Delta \tilde{u}+\lambda\left(\eta_{j}-d_{p}\right)+z(1-\lambda) \frac{\sinh \left(\eta_{j} / z\right)}{\cosh \left(d_{p} / z\right)}, & -d_{p}<\eta_{j}<d_{p}, \\ (j-1) \Delta \tilde{u}+\frac{z e^{d_{p} / z}}{1+e^{2\left(d_{p}-D\right) / z}}\left(e^{\eta_{j} / z}-e^{-\left(\eta_{j}+2 D\right) / z}\right), & -D<\eta_{j}<-d_{p} .\end{cases}
$$

\section{MODEL OF PERISTALTIC LOCOMOTION}

The obtained family of solutions is parametrized by $V$ and incorporates both extension and contraction pulses. To distinguish between the two it is convenient to redefine the half-width as $d=\min \left(d_{p}, D-d_{p}\right)$ and the amplitude as $A=$ $|\tilde{\varepsilon}(0)-\tilde{\varepsilon}(D)|$. The resulting functions $d(V)$ and $A(V)$ are shown in Fig. 3.

The wave train solutions can be used to model peristaltic locomotion $[31,45]$. Suppose that the organism generates a periodic train of extension pulses that propagate rearward with a velocity $V$ so that, when a pulse reaches the tail, another one is initiated at its head with a fixed delay controlled by the parameter $D$. Observations show that when such a pulse moves towards the tail, the latter fattens and gets anchored due to local increase of friction. With such an anchor present, the locomotion naturally occurs into the direction opposite to the direction of the pulse. We mimic such motility pattern in Fig. 4 (left), where the pulse is taken from the range $V<V_{*}$.

Suppose now that the motion of the organism is of stick-slip type with each advance corresponding to passing of a single pulse producing the forward displacement of the head $\Delta \tilde{u}=2 d_{p} \lambda$. Since the next pulse arrives after the time $2 D / V$, the mean translational velocity of the system is $\bar{v}=\Delta \tilde{u} V /(2 D)$. Note that the simultaneous propagation of multiple peristaltic pulses along the animal body is also a possibility, which we do not consider here.

The branch of contraction pulses corresponding to $V>V_{*}$ also generates a motility pattern shown in Fig. 4 (right). In this case the "fattening" and the resulting anchoring takes place around the pulse. Such regimes, driven by passive pulses in otherwise active medium, are however, not realistic due to the necessity to maintain active state throughout the whole body of the organism. 
(a)

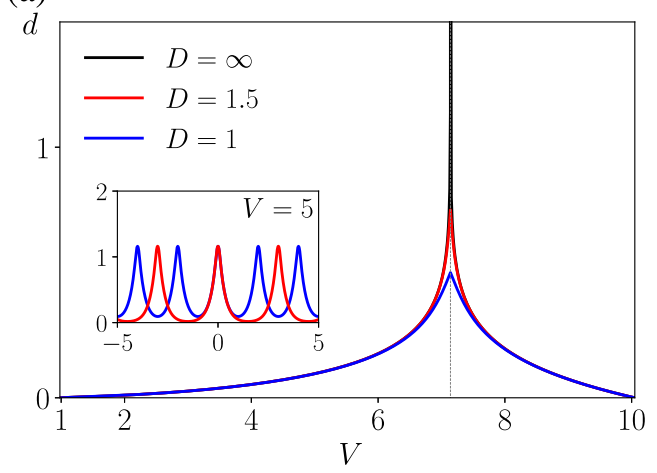

(b)

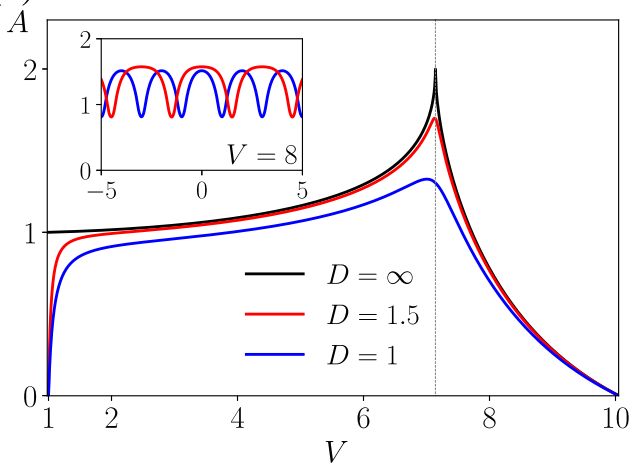

FIG. 3. Trains of active pulses at $\tilde{\sigma}_{a}=100$ : (a) half-width of a single pulse $d(V)$; (b) its amplitude $A(V)$. Vertical asymptotes correspond to $V=V_{*}$; insets illustrate the typical profiles $\tilde{\varepsilon}(\eta)$.

The behavior of the function $\bar{v}(V)$ for both kinds of motility $\left(V \lessgtr V_{*}\right)$ is shown in Fig. 4 (center). We emphasize that around the critical point $V=V_{*}$ the macroscopic velocity $\bar{v}$ behaves singularly: it varies over a broad range around a single value of the control parameter. The corresponding individual pulses take the form of elongated rectangles which is one of the most characteristic features of peristaltic waves. Since the width of these rectangular pulses, and therefore the resulting motility velocity, can vary significantly, being positioned near such critical point can help an organism to adapt its responses. The implied anomalous sensitivity to controls can facilitate optimal behavior in complex physiological conditions and would then be highly functional.

\section{SOLUTION OF THE DISCRETE MODEL}

Given that the biological systems exhibiting peristalsis are usually segmented, the question arises whether our oversimplified (quasi)continuum model (2) adequately represents the dynamics of its discrete prototype (1). To answer this question we now briefly consider the traveling wave solutions of the original discrete system.

We maintain the same normalization and use again the ansatz $\tilde{\varepsilon}_{n}(t)=\tilde{\varepsilon}(\eta)$, where $\eta=(n h-V t) / h$. The discrete strain field $\tilde{\varepsilon}(\eta)$ satisfies the equation

$$
V^{2} \frac{d^{2} \tilde{\varepsilon}}{d \eta^{2}}=\tilde{\sigma}(\eta+1)+\tilde{\sigma}(\eta-1)-2 \tilde{\sigma}(\eta),
$$

with $\tilde{\sigma}(\eta)=\tilde{\varepsilon}(\eta)+\tilde{\sigma}_{a} H(-\eta)$. In view of the partial linearity of the problem, it will be enough to construct the kink-type solution while individual pulses and trains of pulses can be obtained as linear combinations of kinks with appropriately adjusted continuity conditions. As above, the kink solution can be obtained if we use in (20) the ansatz $\tilde{\sigma}(\eta)=\tilde{\varepsilon}(\eta)+$ $\tilde{\sigma}_{a} H(-\eta)$.

Suppose again that a switching point where $\tilde{\varepsilon}(0)=1$ is placed at $\eta=0$. We can then apply the Fourier transform

$$
\hat{\varepsilon}_{k}(k)=\int_{-\infty}^{\infty} \tilde{\varepsilon}_{k}(\eta) e^{i k \eta} d k
$$

and rewrite the problem as an algebraic one:

$$
L(k) \hat{\varepsilon}_{k}(k)=-\tilde{\sigma}_{a} \frac{\omega^{2}(k)}{0+i k},
$$

where the kernel is $L(k)=\omega^{2}(k)-(k V)^{2}$, and

$$
\omega^{2}(k)=4 \sin ^{2}(k / 2),
$$

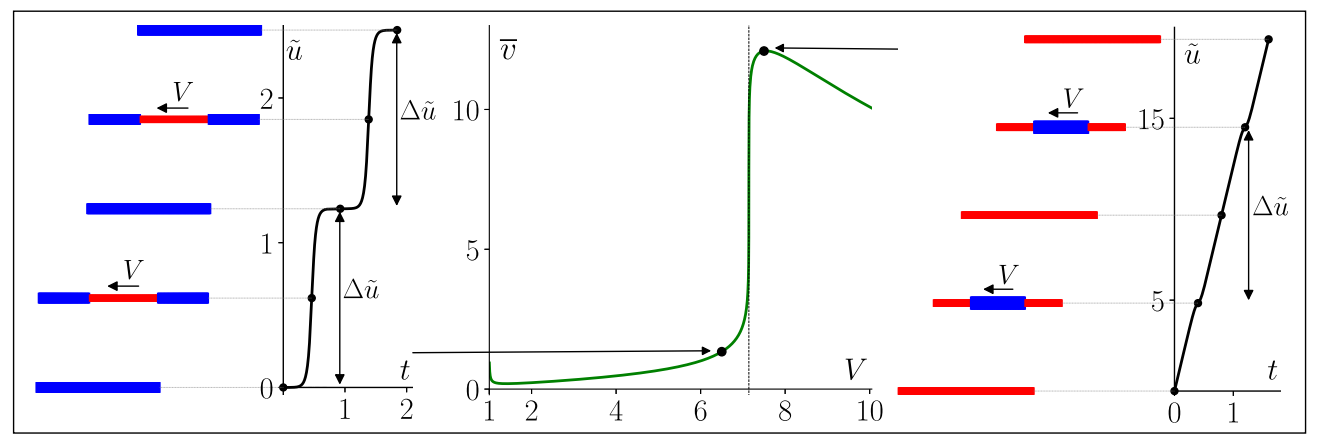

FIG. 4. Schematic presentation of peristalsis by trains of active pulses at $\sigma_{a}=100$ and $D=3$. Motility by extension pulses is shown on the left, and that by contraction pulses is shown on the right. The central plot shows dependence of the average velocity $\bar{v}$ on $V$; the vertical asymptote corresponds to the critical point at $V=V_{*}$. 
is the dispersion relation; the notation $(0+i k)^{-1}=$ $\lim _{\alpha \rightarrow 0+}(\alpha+i k)^{-1}$ stands for the Fourier transform of the Heaviside function.

The solution of the original problem can be presented in the form of the inverse Fourier transform

$$
\tilde{\varepsilon}_{k}(\eta)=\tilde{\varepsilon}_{+}-\frac{\tilde{\sigma}_{a}}{2 \pi} \int_{-\infty}^{\infty} \frac{\omega^{2}(k)}{(0+i k) L(k)} e^{-i k \eta} d k,
$$

where $\tilde{\varepsilon}_{+}=\tilde{\varepsilon}(\infty)$. The contour integral in (22) can be computed by the residue method. The kernel function $L(k)$ has a double zero at $k=0$. The rest of the roots are simple and complex, located in both half-planes. They can be organized in the sets $Z^{ \pm}=\{k: L(k)=0, \pm \operatorname{Im} k>0\}$. Then the explicit series solution of the discrete problem can be written in the form of infinite series:

$$
\tilde{\varepsilon}_{k}(\eta)= \begin{cases}\tilde{\varepsilon}_{+}+\tilde{\sigma}_{a} \sum_{k \in Z^{-}} \frac{\omega^{2}(k)}{k L^{\prime}(k)} e^{-i k \eta}, & \eta>0, \\ \tilde{\varepsilon}_{-}-\tilde{\sigma}_{a} \sum_{k \in Z^{+}} \frac{\omega^{2}(k)}{k L^{\prime}(k)} e^{-i k \eta}, & \eta>0 .\end{cases}
$$

Here $\tilde{\varepsilon}_{+}$is a homogeneous solution of the problem with the boundary conditions $\tilde{\varepsilon}(\eta) \rightarrow \tilde{\varepsilon}_{ \pm}$at $\eta \rightarrow \pm \infty$ and where $\tilde{\varepsilon}_{-}=$ $\tilde{\varepsilon}_{+}+\lambda$.
This solution exists only for the critical value of velocity $V=V_{*}$. In other words, admissible kinks in this model must necessarily satisfy the dynamic Maxwell condition.

To apply the matching condition at $\eta=0$ we need to consider an infinitely large circle in the complex plane. The contour integration in this case gives the relation

$$
\lambda+\tilde{\sigma}_{a} \sum_{k \in Z^{-}} \frac{\omega^{2}(k)}{k L^{\prime}(k)}+\tilde{\sigma}_{a} \sum_{k \in Z^{+}} \frac{\omega^{2}(k)}{k L^{\prime}(k)}=0
$$

If we also recall that $L(-k)=L(k)$ and $L(\bar{k})=\overline{L(k)}$ we obtain $\sum_{k \in Z^{-}} \omega^{2}(k) /\left[k L^{\prime}(k)\right]=\sum_{k \in Z^{+}} \omega^{2}(k) /\left[k L^{\prime}(k)\right]$ and, hence, $\tilde{\varepsilon}(0)=\tilde{\varepsilon}_{+}-\lambda / 2=\tilde{\varepsilon}_{-}+\lambda / 2$. By applying the matching condition $\tilde{\varepsilon}_{ \pm}=1 \mp \lambda$ we recover again the equal area (Maxwell) condition $S_{1}=S_{2}$, saying that the entropy production on the corresponding jump discontinuity in the coarse grained continuum problem is equal to zero.

We can also reconstruct the discrete displacement field by inverting the relation $\left(e^{-i k}-1\right) \hat{u}_{k}(k)=\hat{\varepsilon}_{k}(k)$. Following the same scheme as above we get

$$
\tilde{u}_{k}(\eta)= \begin{cases}\tilde{\varepsilon}_{+}(\eta-1 / 2)+\tilde{\sigma}_{a} \sum_{k \in Z^{-}} \frac{i \omega^{2}(k)}{k \sin (k / 2) L^{\prime}(k)} e^{-i k(\eta-1 / 2)}, & \eta>1 / 2, \\ \tilde{\varepsilon}_{-}(\eta-1 / 2)-\tilde{\sigma}_{a} \sum_{k \in Z^{+}} \frac{i \omega^{2}(k)}{k \sin (k / 2) L^{\prime}(k)} e^{-i k(\eta-1 / 2)}, & \eta<1 / 2 .\end{cases}
$$

Using (23) and (25) we can also obtain the solution describing extension pulses. In this case we must solve (20) with the stress ansatz $\tilde{\sigma}(\eta)=\tilde{\varepsilon}(\eta)+\tilde{\sigma}_{a} \operatorname{rect}(\eta /(2 d))$ and use the same matching condition $\tilde{\varepsilon}( \pm d)=1$. The linearity of the system at fixed $d$ suggests again that the solitary wave solution can be obtained as a linear combination of two kinks $\tilde{\varepsilon}(\eta)=\tilde{\varepsilon}_{k}(\eta-d)-\tilde{\varepsilon}_{k}(\eta+d)$, where the nonlinear relation
$d=d(V)$ is to be found from the relation $\tilde{\varepsilon}_{k}(0)-\tilde{\varepsilon}_{k}(2 d)=$ 1. The corresponding solution for the discrete strain field is $\tilde{u}(\eta)=\tilde{u}_{k}(\eta-d)-\tilde{u}_{k}(\eta+d)$, which can be obtained by inverting the equation $\left(e^{-i k}-1\right) \hat{u}(k)=\hat{\varepsilon}(k)$ in the Fourier space. The total displacement which is again $\Delta \tilde{u}=2 d \lambda$.

The typical dependencies $d(V)$ and $A(V)$ for extension pulses are illustrated in Fig. 5, where they are compared with (a)

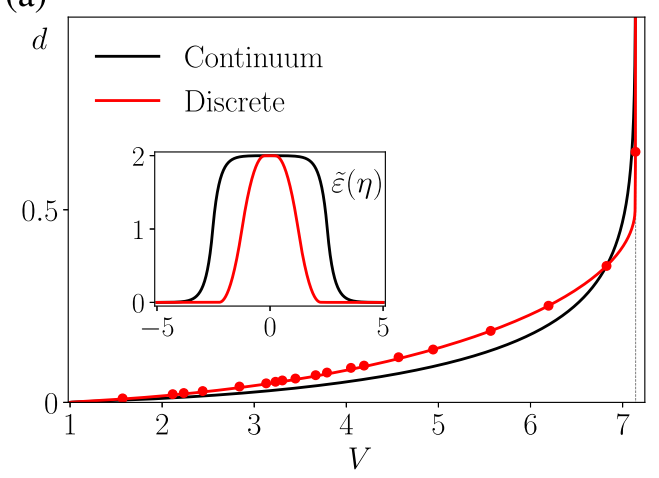

(b)

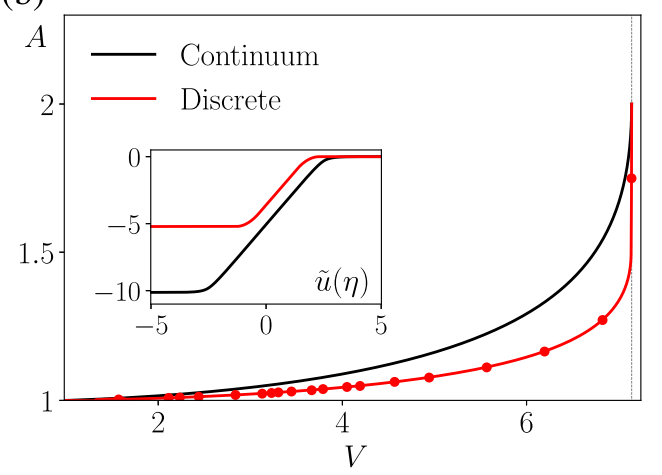

FIG. 5. Comparison of the predictions of discrete (red) and continuum (black) models at $\tilde{\sigma}_{a}=100$ : (a) half-width, (b) amplitude. The red markers show the discrete pulses obtained numerically by solving a one-parametric set of initial value problems; see Fig. 6 . The insets compare discrete and continuum distributions of strains (a) and displacements (b) at the near critical speed $V=V_{*}-10^{-7}$. 


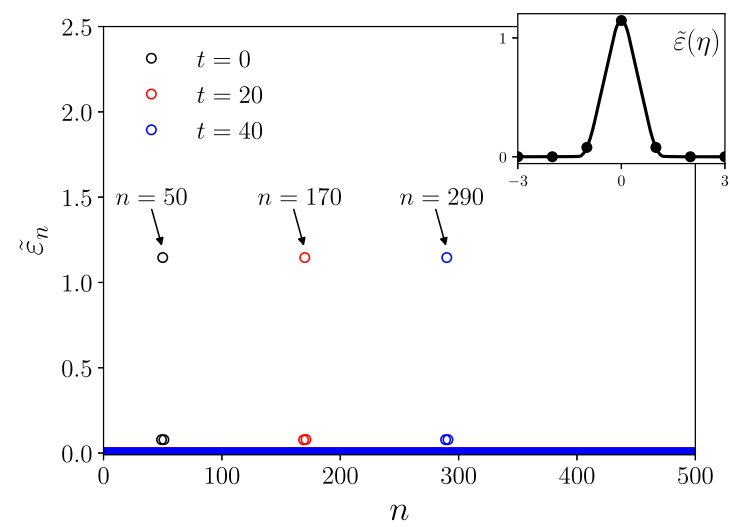

FIG. 6. Stable propagation of an extension pulse at $V=6$ and $\tilde{\sigma}_{a}=100$. The inset shows the superimposed analytical solution.

the corresponding results of the continuum theory with $h=1$; the comparison confirms the overall adequacy of the continuum approximation despite the choice of a finite value for our "small" parameter. The discrete dots in Fig. 5 also overlap exactly with our numerical computations illustrated in Fig. 6 where we tested the stability of the obtained traveling wave solutions by using them as initial data and solving numerically the corresponding initial value problem.

For the contraction pulse we need to use another ansatz, $\tilde{\sigma}(\eta)=\tilde{\varepsilon}(\eta)+\tilde{\sigma}_{a}[1-\operatorname{rect}(\eta /(2 d))]$. The discrete strain field is then $\tilde{\varepsilon}(\eta)=\tilde{\varepsilon}_{k}(d+\eta)+\tilde{\varepsilon}_{k}(d-\eta)$, while the displacement field is $\tilde{u}(\eta)=\tilde{u}_{k}(d+\eta)+\tilde{u}_{k}(d-\eta)$. The value of the parameter $d$ in the corresponding interval of velocities $V_{*}<$ $V \leqslant V_{* *}$ can be found from the equation $\tilde{\varepsilon}_{k}(0)+\tilde{\varepsilon}_{k}(2 d)=1$.

Using the same idea we can find solutions describing trains of pulses. In the case of extension we need to use the ansatz $\tilde{\sigma}(\eta)=\tilde{\varepsilon}(\eta)+\sum_{j} \tilde{\sigma}_{a} \operatorname{rect}\left(\eta_{j} /\left(2 d_{p}\right)\right)$. The strain and displacement fields are now represented via infinite sums, $\tilde{\varepsilon}(\eta)=$ $\sum_{j} \tilde{\varepsilon}_{k}\left(\eta_{j}-d_{p}\right)-\tilde{\varepsilon}_{k}\left(\eta_{j}+d_{p}\right)$ and $\tilde{u}(\eta)=\sum_{j} \tilde{u}_{k}\left(\eta_{j}-d_{p}\right)-$ $\tilde{u}_{k}\left(\eta_{j}+d_{p}\right)$, respectively. The equation for finding $d_{p}$ takes the form

$$
\sum_{j} \tilde{\varepsilon}_{k}(-2 j D)-\tilde{\varepsilon}_{k}\left(2 d_{p}-2 j D\right)=1 .
$$

In the discrete case we can also define the parameter $d=$ $\min \left(d_{p}, D-d_{p}\right)$, observing that $d_{p}=D / 2$ at $V=V_{*}$.

We tested the numerical stability of the obtained discrete pulses by solving a range of initial value problems. The stable propagation of a triangular discrete extension pulse is illustrated in Fig. 6, where we considered a discrete chain with 500 springs. A more relevant for peristalsis example of a stable propagation of a rectangular extension pulse is shown in Fig. 7(a) for the chain with 1000 springs. In this test we used initial conditions $\tilde{u}_{n}(0)=0, \dot{\tilde{u}}_{1}(0)=v_{0}$, and $\dot{\tilde{u}}_{n}(0)=$ $0, n>1$, and assumed free ends. The time evolution of the displacement field, shown in Fig. 7(b), illustrates the creation of the finite displacement behind the propagating pulse.

\section{CONCLUSIONS}

We developed a model of a dynamic passive-to-active transformation taking place in the front of a steadily moving pulse with the corresponding reverse transformation taking place in its rear. The associated solitary wave solutions were extended as periodic trains and used to model the peristaltic mode of self propulsion. We found that at the critical value of parameter the model generates singular regimes with diverging effective correlation length and argued that such criticality may be functional. Our results can be used for biomimetic reproduction of wormlike motion in applications ranging from endoscopic diagnostics [46] to pipeline inspection [47]. While in the existing robotic systems activity is imitated by globally synchronized distributed actuators [9,20,36], our focus on local mechanical feedback in muscle-type soft materials (a)

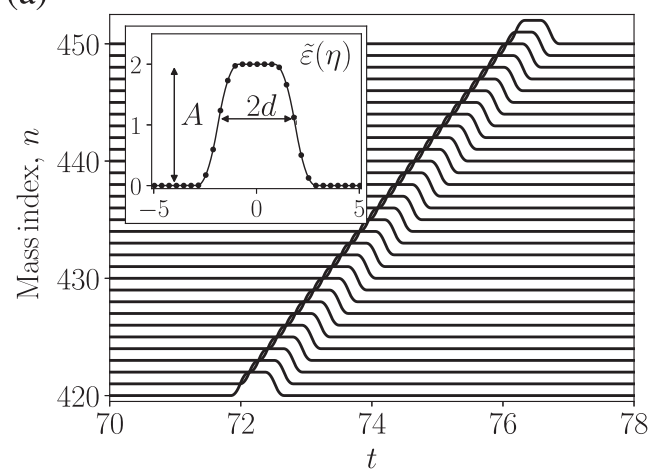

(b)

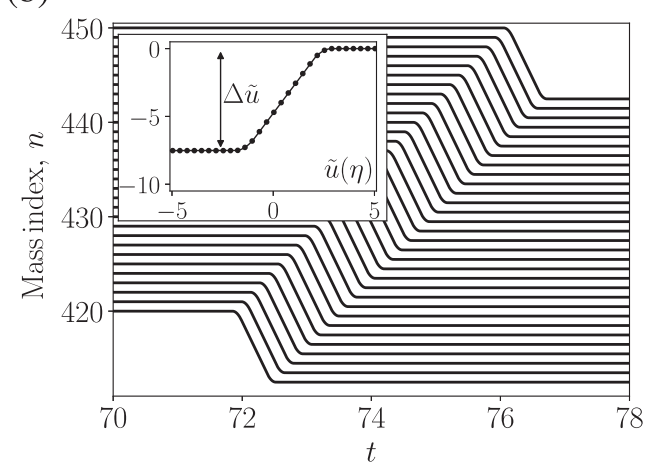

FIG. 7. Results of numerical experiments with discrete chain at $\tilde{\sigma}_{a}=100$ and $v_{0}=200$ (see the text): (a) strains, (b) displacements. The generated extension pulse correspond to $V \approx V_{*}, d \approx 1.88$, and $A \approx 1.998$. Insets show the comparison of the numerical solution in the comoving frame (markers) with the corresponding analytical solution (solid line). 
opens new avenues in the biomimetic modeling of peristalsis, see also [21,48,49]. The proposed prototypical model can serve only as a proof of concept and future work aimed at quantitative predictions, should incorporate energy supply and dissipation while also accounting for realistic threedimensional geometry.

\section{ACKNOWLEDGMENTS}

The authors thank J. F. Joanny, G. Mishuris, P. Recho, and A. Vainchtein for helpful discussions and acknowledge the support of the French Agence Nationale de la Recherche under Grant No. ANR-17-CE08-0047-02.
[1] M. D. Sinnott, P. W. Cleary, and S. M. Harrison, Appl. Math. Model. 44, 143 (2017).

[2] S. Brandstaeter, S. L. Fuchs, R. C. Aydin, and C. J. Cyron, GAMM-Mitteilungen 42, e201900001 (2019).

[3] J. Gray and H. Lissmann, J. Exp. Biol. 15, 506 (1938).

[4] G. Chapman, Biol. Rev. 33, 338 (1958).

[5] H. Jones, J. Zool. 186, 407 (1978).

[6] R. M. Alexander, Locomotion of Animals, Vol. 163 (Blackie, Glasgow, 1982).

[7] K. J. Quillin, J. Exp. Biol. 202, 661 (1999).

[8] T. Tyrakowski, P. Kaczorowski, W. Pawłowicz, M. Ziółkowski, P. Smuszkiewicz, I. Trojanowska, A. Marszaek, M. Żebrowska, M. Lutowska, E. Kopczyńska et al., Folia Biol. 60, 99 (2012).

[9] D. Agostinelli, F. Alouges, and A. DeSimone, Front. Rob. AI 5, 99 (2018).

[10] J. H. Boyle, S. Berri, and N. Cohen, Front. Comput. Neurosci. 6, 10 (2012).

[11] K. A. Daltorio, A. S. Boxerbaum, A. D. Horchler, K. M. Shaw, H. J. Chiel, and R. D. Quinn, Bioinspiration Biomimetics 8, 035003 (2013).

[12] J. H. Boyle, S. Johnson, and A. A. Dehghani-Sanij, IEEE/ASME Trans. Mechatronics 18, 439 (2012).

[13] G. Kastberger, T. Hoetzl, M. Maurer, I. Kranner, S. Weiss, and F. Weihmann, PLoS ONE 9, e86315 (2014).

[14] J. H. Cartwright, Europhys. News 37, 22 (2006).

[15] A. P. Solon, J.-B. Caussin, D. Bartolo, H. Chaté, and J. Tailleur, Phys. Rev. E 92, 062111 (2015).

[16] W. Ngamsaad and S. Suantai, Phys. Rev. E 98, 062618 (2018).

[17] O. A. Dudchenko and G. T. Guria, Phys. Rev. E 85, 020902(R) (2012).

[18] A. J. Ijspeert, Neural Networks 21, 642 (2008).

[19] P. Paoletti and L. Mahadevan, Proc. R. Soc. B 281, 20141092 (2014).

[20] P. W. Miller and J. Dunkel, Soft Matter 16, 3991 (2020).

[21] C. Pehlevan, P. Paoletti, and L. Mahadevan, Elife 5, e11031 (2016).

[22] J. Prost, F. Jülicher, and J.-F. Joanny, Nat. Phys. 11, 111 (2015).

[23] F. Jülicher, S. W. Grill, and G. Salbreux, Rep. Prog. Phys. 81, 076601 (2018).

[24] R. J. Hawkins and T. B. Liverpool, Phys. Rev. Lett. 113, 028102 (2014).

[25] A. Maitra and S. Ramaswamy, Phys. Rev. Lett. 123, 238001 (2019).

[26] M. Moshe, M. J. Bowick, and M. C. Marchetti, Phys. Rev. Lett. 120, 268105 (2018).
[27] C. Scheibner, A. Souslov, D. Banerjee, P. Surówka, W. T. M. Irvine, and V. Vitelli, Nature Phys. 16, 475 (2020).

[28] J. R. Raney, N. Nadkarni, C. Daraio, D. M. Kochmann, J. A. Lewis, and K. Bertoldi, Proc. Natl. Acad. Sci. USA 113, 9722 (2016).

[29] N. Nadkarni, C. Daraio, and D. M. Kochmann, Phys. Rev. E 90, 023204 (2014).

[30] B. Deng, L. Chen, D. Wei, V. Tournat, and K. Bertoldi, Sci. Adv. 6, eaaz1166 (2020).

[31] Y. Tanaka, K. Ito, T. Nakagaki, and R. Kobayashi, J. R. Soc. Interface 9, 222 (2012).

[32] A. Kandhari, Y. Wang, H. J. Chiel, and K. A. Daltorio, Soft Robotics 6, 560 (2019).

[33] J. B. Keller and M. S. Falkovitz, J. Theor. Biol. 104, 417 (1983).

[34] M. Wadepuhl and W.-J. Beyn, J. Theor. Biol. 136, 379 (1989).

[35] H. Fang, S. Li, K. Wang, and J. Xu, Bioinspiration Biomimetics 10, 066006 (2015).

[36] Z. Jiang and J. Xu, Micromachines 8, 364 (2017).

[37] M. Badoual, F. Jülicher, and J. Prost, Proc. Natl. Acad. Sci. USA 99, 6696 (2002).

[38] X. Serra-Picamal, V. Conte, R. Vincent, E. Anon, D. T. Tambe, E. Bazellieres, J. P. Butler, J. J. Fredberg, and X. Trepat, Nat. Phys. 8, 628 (2012).

[39] P. Rosenau, Phys. Lett. A 118, 222 (1986).

[40] L. Truskinovsky and A. Vainchtein, Continuum Mech. Thermodyn. 18, 1 (2006).

[41] L. R. Gómez, A. M. Turner, and V. Vitelli, Phys. Rev. E 86, 041302 (2012).

[42] V. Nesterenko, Dynamics of Heterogeneous Materials (Springer, New York, 2013).

[43] L. M. Truskinovsky, Dokl. Akad. Nauk 265, 306 (1982).

[44] J.-B. Caussin, A. Solon, A. Peshkov, H. Chaté, T. Dauxois, J. Tailleur, V. Vitelli, and D. Bartolo, Phys. Rev. Lett. 112, 148102 (2014).

[45] E. G. Hemingway and O. M. O'Reilly, Biomech. Model. Mechanobiol. 20, 5 (2021).

[46] C. Stefanini, A. Menciassi, and P. Dario, Int. J. Robotics Res. 25, 551 (2006).

[47] A. Yamashita, K. Matsui, R. Kawanishi, T. Kaneko, T. Murakami, H. Omori, T. Nakamura, and H. Asama, in 2011 IEEE International Conference on Robotics and Biomimetics (IEEE, Piscataway, NJ, 2011), pp. 1017-1023.

[48] T. Umedachi, T. Kano, A. Ishiguro, and B. A. Trimmer, R. Soc. Open Sci. 3, 160766 (2016).

[49] A. DeSimone, in The Mathematics of Mechanobiology (Springer, Cham, 2020), pp. 1-41. 